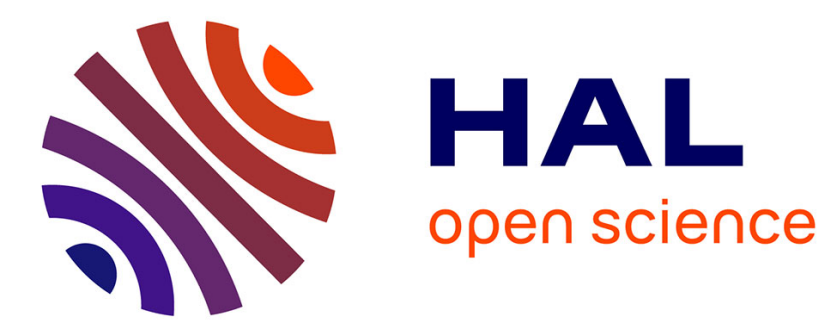

\title{
Target 5G visible light positioning signal subcarrier extraction method using particle swarm optimization algorithm
}

\author{
Yanqi Huang, Xun Zhang, El Hassane Aglzim, Lina Shi
}

\section{- To cite this version:}

Yanqi Huang, Xun Zhang, El Hassane Aglzim, Lina Shi. Target 5G visible light positioning signal subcarrier extraction method using particle swarm optimization algorithm. IEEE International Symposium on Broadband Multimedia Systems and Broadcasting, Aug 2021, Chengdu, China. hal03272714

\author{
HAL Id: hal-03272714 \\ https://hal.science/hal-03272714
}

Submitted on 28 Jun 2021

HAL is a multi-disciplinary open access archive for the deposit and dissemination of scientific research documents, whether they are published or not. The documents may come from teaching and research institutions in France or abroad, or from public or private research centers.
L'archive ouverte pluridisciplinaire HAL, est destinée au dépôt et à la diffusion de documents scientifiques de niveau recherche, publiés ou non, émanant des établissements d'enseignement et de recherche français ou étrangers, des laboratoires publics ou privés. 


\section{Target 5G visible light positioning signal subcarrier extraction method using particle swarm optimization algorithm}

\author{
$1^{\text {st }}$ Yanqi Huang \\ DRIVE EA1859, \\ Univ. Bourgogne Franche Comté \\ Institut superieur d'electronique de Paris \\ France \\ Yanqi_Huang@etu.u-bourgogne.fr \\ $2^{\text {nd }}$ Xun Zhang \\ Institut superieur d'electronique de Paris \\ Paris, France \\ xun.zhang@isep.fr \\ $4^{\text {th }}$ Lina Shi \\ Laboratoire d'Ingénierie des \\ Systèmes de Versailles, Université de Versailles \\ Saint-Quentin-en-Yvelines \\ Vélizy, France \\ linashi@lisv.uvsq.fr
}

\author{
$3^{\text {rd }}$ El-Hassane Aglzim \\ DRIVE EA1859, \\ Univ. Bourgogne Franche Comté \\ Nevers, France \\ el-hassane.aglzim@u-bourgogne.fr
}

\begin{abstract}
With the explosive growth of demand for Internet of Things (IoT) applications and the increasing dependence of users on wireless connections, indoor location based service(LBS) under 5G-Public-Private Partnership (5G-PPP) using cases have received more attention and get rapid development. Thanks to the safty, security and customization of 5G network pointed by $5 \mathrm{G}$ forum white paper, indoor positioning systems using unified 5G New Radio (NR) signals have become the focus of the next generation of visible light positioning (VLP) systems. In 5G New Radio(NR) frame, totally 192 subcarriers are used to carry positioning reference signal(PRS). In order to improve the position accuracy, the aim of this study is to investigate the method of the 5G NR subcarrier selection. A certain number of PRSs are obtained from the signal source of 192 subcarriers, and the minimum positioning error(PE) is calculated by using particle swarm optimization(PSO) algorithm. Then the relationship between the PRS with the minimum PE and the position of the subcarrier is analyzed which achieved the subcarrier extraction. The experimental results show that the average $P E$ of this scheme is below $6 \mathrm{~cm}$, and the minimum $P E$ can reach $0.05 \mathrm{~cm}$. This approach is confirmed the feasibility of high accuracy in 5G indoor VLP application and has the potential to be extended to larger and more complex 5G-PPP scenarios.

Index Terms-5G, Visible Light Positioning(VLP), Visible Light Communication(VLC), Received Signal Strength Indication(RSSI), Localization, particle swarm optimization(PSO)
\end{abstract}

\section{INTRODUCTION}

With the rapid expansion of Internet of Things(IoT) applications and stronger user dependence on wireless connections, there has been a growing interest in the possibilities and applications using 5G. There are 6 families of using cases of 5G-Public-Private Partnership(5G-PPP) and more than 30 use cases were proposed in white paper of 5G PPP use cases and performance evaluation models [1]. Specifically, the robots used to diagnose eHealth play one of the potential applications in vertical industry business cases. These are expected to be used in assisted living remote surgery and cloud service robots. In the process of designing and improving the performance of diagnostic eHealth robotics in indoor area, the location information of robotics has stronger user dependence, thus Location Based Service(LBS) has received more attention and get rapid development. The research in this topic is conducted in the European H2020 project Internet of Radio Light(IoRL), which was the first to propose a hybrid indoor optical-radio network in convergence with the 5G Era [2]-[4].

Visible light positioning(VLP) is considered as the most promising candidate of $5 \mathrm{G}$ indoor LBS because of many attractive features such as high level of security [5], long system lifetime [6], no radio frequency interference [7] and low cost [8]. VLP system uses the received signal as positioning reference signal(PRS), the input of the position system, to calculate the receiver's position. Recently, researches about how to establish and improve the performance of $5 \mathrm{G}$ indoor VLP system has a significant increase. Research [9] proposed a location-based broadcasing application for museum scenarios based on 5G IoRL infrastructure, and gave a demonstration setup in detail. Research [10] focused on improving vertical and horizontal localization for use case scenarios and targeted to enhance the learning of network signal behavior. Research [11] proposed a 5G indoor localization method using the data fusion of Time Difference of Arrival(TDOA) and Pedestrian Dead Reckoning(PDR), and solve the limitation of the base station selection problem. Research [12] presented a multilayer network architecture by integrating visible light communication(VLC) and VLP within 5G networks, in order to guarantee the diverse IoT requirements. In study [13], a device identification method integrates VLC-based communication, 


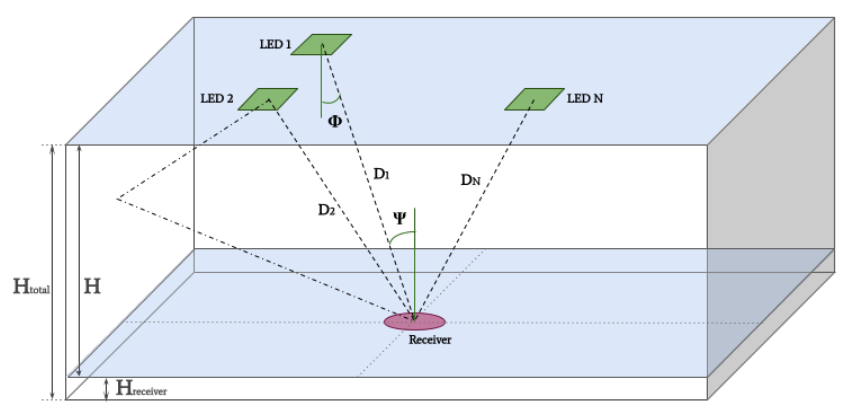

Fig. 1. 5G Indoor positioning system scenario

positioning and illumination systems within the $5 \mathrm{G}$ network was proposed.

In order to adapt the intensity modulation and direct detection(IM/DD) and keep a high spectrum efficient in VLC , the orthogonal frequency-division multiplexing (OFDM)based modulation schemes are widely used to generate the real and non-negative positioning signals. In 5G New Radio(NR) frame of IoRL, a total of 192 subcarriers are used to carry PRSs. If we regard the PRSs in a same timestamp as a measurement, as the received signal power is related to the frequency of the signal [14], PRSs received by different subcarriers will be different in the same measurement. That is to say, if PRSs carried by 5G NR signal are considered as inputs of Received Signal Strength Indication(RSSI)-based VLP system, it is necessary to extract the subcarriers with the minimum positioning error.

However, few researchers have addressed the signal extraction method in the midst of $5 \mathrm{G}$ NR signal sources. In this paper, we used PRS values from 192 subcarriers to calculate the positioning error in $K$ receiver's reference points. Then we recorded PRSs with the minimum positioning error, and also recorded its corresponding subcarrier sequence number. After this process was implemented in $K$ receiver's reference points, we imported all these PRSs and its subcarrier positions into a multi-Support Vector Machine(SVM) to build a subcarrier prediction model. Once we establish this model, we can get the predict subcarrier sequence number we should use for positioning when we measure a set of PRSs. The experimental results verify that by extracting subcarriers from $5 \mathrm{G}$ NR signal sources, the positioning error was reduced by $73.28 \%$. In other words, our proposal can give a solution of how to extraction subcarriers from $5 \mathrm{G}$ NR sources.

The organization of this paper is as follows. Section II describes the 5G VLP system model and introduce the format of $5 \mathrm{G}$ positioning reference signal. Section III describles the detail of the extraction method of PRSs. Section IV gives the experimental results and analysis with details of the experiment testbed. Section V gives the conclusions.

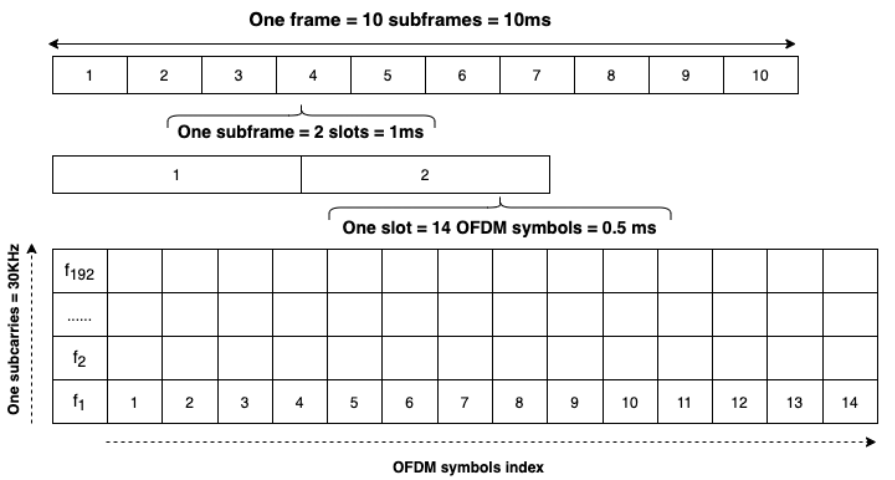

Fig. 2. The structure of 5G NR signal

\section{5G RSSI-BASED VLP SYSTEM}

\section{A. 5G VLP System overview}

Fig. 1 shows a generic 5G indoor positioning system scenario. Several LEDs are used as transmitters. The target is equipped with an Avalanche Photodiode (APD) as a receiver. Traditional RSSI-based VLP system uses received PRSs as RSSI to calculate transmitter-receiver distances and estimate the coordinate of receiver. Here, assumed that there are $N(N \geq 3)$ LEDs at the same height and all LEDs follow the Lambertian distribution and there are $S(S \geq 2)$ subcarriers.

\section{B. The file format of PRS}

The structure of $5 \mathrm{G}$ NR signal modulated by OFDM is illustrated in Fig.2. For each frame there are 10 subframes, and for each subframe there are 2 slots. Since one time slot duration is $0.5 \mathrm{~ms}$, each PRS data is obtained by user equipment every $36 \mu \mathrm{s}(0.5 \mathrm{~ms} / 14)$ at least. We set every 36 $\mu$ s as a measurement timestamp.

Fig. 3 shows the file format of PRS format exported by user equipment. Each line in the file represents one measurement timestamp, and it is followed by $S$ PRSs on its subcarriers. In other words, for $T$ measurements, totally $T \times S$ PRSs are recorded on user equipment. The method of selecting the PRSs among $S$ subcarriers will be described in the next section.

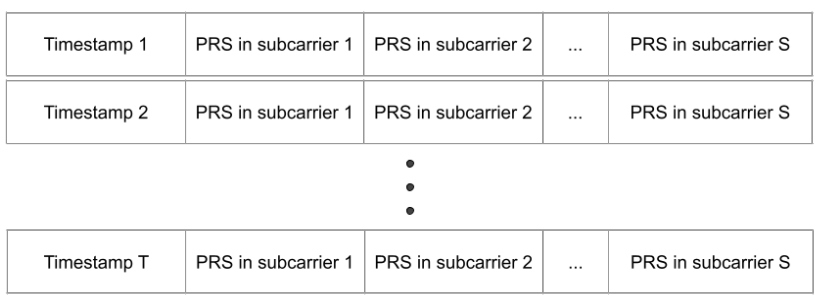

Fig. 3. File format of PRS exported by UE

\section{EXTRACTION OF PRSS}

The process of the proposal contains two phases: offline and online. In offline, a set of environmental indexes is used to describe the environmental disturbances in each subcarrier, 
and to calculate $\mathrm{PE}$ together with the $\mathrm{Pr}$ on each subcarrier. The objective of offline is to record the subcarrier number $s$ that can obtain the smallest PE, and the objective of online is to extraction the PRS in subcarrier $s$ for localization.

\section{A. The offline phase}

Received signal power in RSSI-based VLP system has been proved to be related to the frequency of the signal [14]. By using the received signal power of each subcarrier to estimate the coordinates of the receiver, the subcarrier that can obtain the minimum $\mathrm{PE}$ is the subcarrier suitable for positioning. To describe environment characteristics of all subcarriers $S$

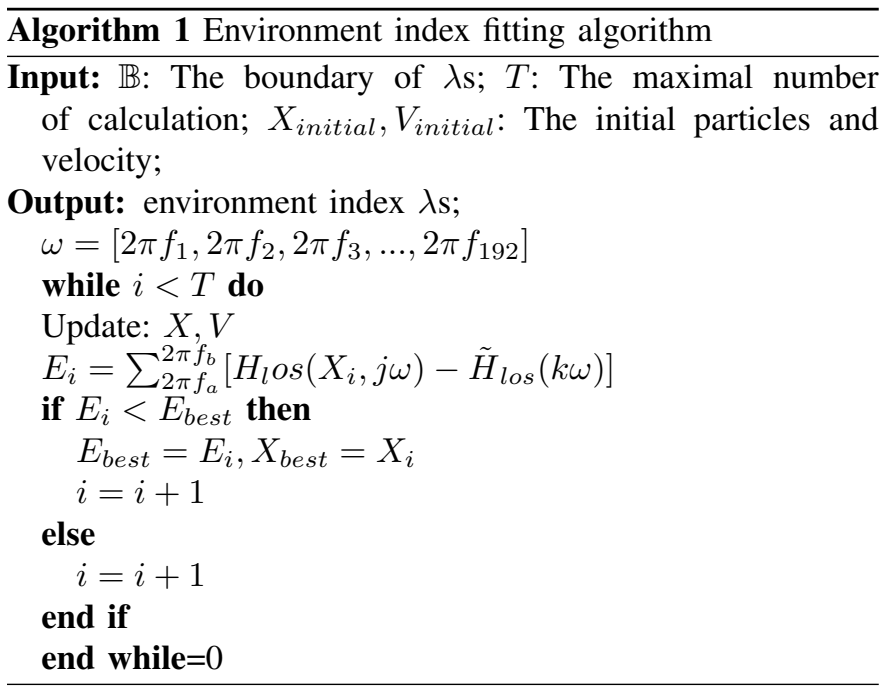

for LED $x(x \in[1, N]), m$ and $G$ are used to represent the illumination and circuit environment respectively, and we let $\lambda=\{m, G, s\}$. To simplify the illustration, the process of environment index $\lambda_{x y s}$ extraction in subcarrier $s, s \in[1, S]$ on $y$ th $(y \in[1, K])$ reference point for LED $x$ is detailed as an example. The estimated distance $\left(\hat{D_{x y s}}\right)$ was calculated by received PRSs power. Based on $n$ pairs of estimated and realistic distances $\left(D_{x y s}\right)$, an optimization algorithm PSO can be used to fit the environment index $\lambda_{x y}$ shown by equation (1) (2) and pseudocode of this algorithm is given in Algorithm.1. The environment index $\lambda$ consists of three parameters (equation (3)), where $m, G$ and $s$ represent the compensation of Lambertian distribution, the extra pathloss of circuit and the number of subcarrier which can cause the minimum PE.

$$
\begin{gathered}
\hat{D}_{x y s}=G_{x y s}\left(\frac{A\left(m_{0}+m_{x y s}+1\right) P_{t_{-} s} H^{\left(m_{0}+m_{x y s}+1\right)}}{2 \pi P_{r_{-} s}}\right)^{\frac{1}{m_{0}+m_{x y s}+3}} \\
\min _{\lambda_{x y s}} \sum_{i=1}^{n}\left[D_{x y s}-\hat{D_{x y s}}\left(P_{r_{-} s}\right)\right] \\
\lambda=\{m, G, s\}
\end{gathered}
$$

Once environment indexes of all LEDs on all reference points have been extracted completely, the sets $\Lambda$ and $P$ (equations (4) (5)) are obtained and applied to train the classification model. This model builds up the mapping between environment indexes and the PRSs power shown in (6). In this paper, an one-against-all Multi-SVM classifier [15] has been employed.

$$
\begin{aligned}
\Lambda & =\left\{\begin{array}{cccc}
\lambda_{11} & \lambda_{12} & \ldots & \lambda_{1 K} \\
\lambda_{21} & \ldots & \ldots & \\
\ldots & \ldots & \ldots & \\
\lambda_{N 1} & \ldots & \ldots & \lambda_{N K}
\end{array}\right\} \\
P & =\left\{\begin{array}{cccc}
P_{11} & P_{12} & \ldots & P_{1 K} \\
P_{21} & \ldots & \ldots & \\
\ldots & \ldots & \ldots & \\
P_{N 1} & \ldots & \ldots & P_{N K}
\end{array}\right\} \\
M: P \rightarrow \Lambda &
\end{aligned}
$$

\section{B. The online phase}

The objective of this phase is to estimate the coordinates of the target receiver. When the receiver captures real-time PRSs powers of each LED, the corresponding environment indexes $\hat{\lambda}$ will be predicted by the classification model $M$ shown in equation (7). As the distances between receiver and each LED have been figured out, the set of distances $\hat{D}$ is used to estimate the coordinate of the receiver $(\hat{x}, \hat{y}, \hat{z})$ by $\mathrm{RSSI}$ trilateration, shown in equations (8) to (13).

$$
M\left(\left[P_{r}^{1}, P_{r}^{2}, \ldots, P_{r}^{N}\right]\right) \rightarrow \hat{\lambda}(\hat{m}, \hat{G}, \hat{s})
$$

$\left\{\hat{D} \mid \hat{D}_{i}=\hat{G}^{i}\left(\frac{A\left(m_{0}+\hat{m}^{i}+1\right) P_{t_{-} s} H^{\left(m_{0}+\hat{m}^{i}+1\right)}}{2 \pi P_{r_{-} s}}\right)^{\frac{1}{m_{0}+\hat{m}^{i}+3}}, i \in[3, N]\right\}$

$$
\left\{\begin{array}{c}
\left(x_{1}-\hat{x}\right)^{2}+\left(y_{1}-\hat{y}\right)^{2}+\left(z_{1}-\hat{z}\right)^{2}=\hat{D}_{1} \\
\left(x_{2}-\hat{x}\right)^{2}+\left(y_{2}-\hat{y}\right)^{2}+\left(z_{2}-\hat{z}\right)^{2}=\hat{D}_{2} \\
\vdots \\
\left(x_{N}-\hat{x}\right)^{2}+\left(y_{N}-\hat{y}\right)^{2}+\left(z_{N}-\hat{z}\right)^{2}=\hat{D_{N}}
\end{array}\right.
$$

where

$$
X=\left[\begin{array}{l}
\hat{x} \\
\hat{y} \\
\hat{z}
\end{array}\right]
$$

$$
\begin{gathered}
A=\left[\begin{array}{ccc}
2 x_{2}-2 x_{1} & 2 y_{2}-2 y_{1} & 2 z_{2}-2 z_{1} \\
2 x_{3}-2 x_{1} & 2 y_{3}-2 y_{1} & 2 z_{3}-2 z_{1} \\
\vdots & \vdots & \vdots \\
2 x_{N}-2 x_{1} & 2 y_{N}-2 y_{1} & 2 z_{N}-2 z_{1}
\end{array}\right] \\
B=\left[\begin{array}{c}
\hat{D}_{1}^{2}-\hat{D}_{2}^{2}-x_{1}^{2}+x_{2}^{2}-y_{1}^{2}+y_{2}^{2}-z_{1}^{2}+z_{2}^{2} \\
\hat{D}_{1}^{2}-\hat{D}_{3}^{2}-x_{1}^{2}+x_{3}^{2}-y_{1}^{2}+y_{3}^{2}-z_{1}^{2}+z_{3}^{2} \\
\vdots \\
\hat{D}_{1}^{2}-\hat{D}_{N}^{2}-x_{1}^{2}+x_{N}^{2}-y_{1}^{2}+y_{N}^{2}-z_{1}^{2}+z_{N}^{2}
\end{array}\right]
\end{gathered}
$$




$$
\left[\begin{array}{l}
\hat{x} \\
\hat{y} \\
\hat{z}
\end{array}\right]=\left(A^{T} A^{-1}\right) A^{T} B
$$

where $\left(x_{i}, y_{i}, z_{i}\right)$ represents the coordinate of $i t h$ LED. To evaluate the positioning accuracy, the positioning error is defined as equation (14)

$$
P E=\sqrt{(\hat{x}-x)^{2}+(\hat{y}-y)^{2}+(\hat{z}-z)^{2}}
$$

\section{EXPERIMENT RESULTS AND ANALYSIS}

\section{A. Setup of testbed}

For 5G VLP system, the flow chart of the experiment is shown in 4(a). A server (Dell R740) acts as the 5G new radio(NR) transmitter-generated $5 \mathrm{G}$ baseband signal. It modulates the user data with different modulation schemes which include QPSK, 16-QAM, 64-QAM and 256-QAM. As the modulated symbols were assigned to the $5 \mathrm{G}$ NR frame shown in Fig.2, inverse Fast Fourier Transform (IFFT) processing and IQ modulation were applied to convert the OFDM frame to the time domain and guarantee the signal well matched with the IM/DD VLC system. Subsequently, a digital-analog (D/A) converter (USRP 2944R) and a DC bias transformed this digital signal to analog signal with an appropriate DC component. This signal is sent to four LEDs (LUXEN 5050). Through an optical wireless channel, the signal from each LED is captured by a commercial receiver (HAM C5331-11) in sequence and digitized by an analog-digital (A/D) converter (USRP 2944R). Finally, the received signal is transferred back to the location server.

The setup of experimental is shown in 4(b). Four LEDs were fixed on the ceiling in a known location. In reality, receiver is not always placed on the ground. We did two measurement when the height of the receiver is $1.042 \mathrm{~m}$, and compared the positioning result with the height of $0.272 \mathrm{~m}$. For each of them, the receiver moved on a plane parallel to the ground. The distribution of the EVM (Error Vector Magnitude) of each LED in the whole area was tested to describe the optical wireless channel. Subsequently, signal samples from each LED were collected 10 times by the receiver at 24 different points. The parameters of the experiment are illustrated in Table. I.

\section{B. Experiment results and analysis}

In this part, we compared the positioning errors of two methods: our proposal and the method without subcarrier extraction. The latter did not extract specific subcarriers for positioning, but took the average values of PRSs in 192 subcarriers. The positioning errors of two methods are presented in Table.II.

Fig.5 and Fig.6 respectively show distributions of reference points and test points of two method. The real locations of the receivers are represented as blue points and their estimated locations are represented as a orange points. In Fig.5, the average positioning error of estimated points was $22.34 \mathrm{~cm}$, and

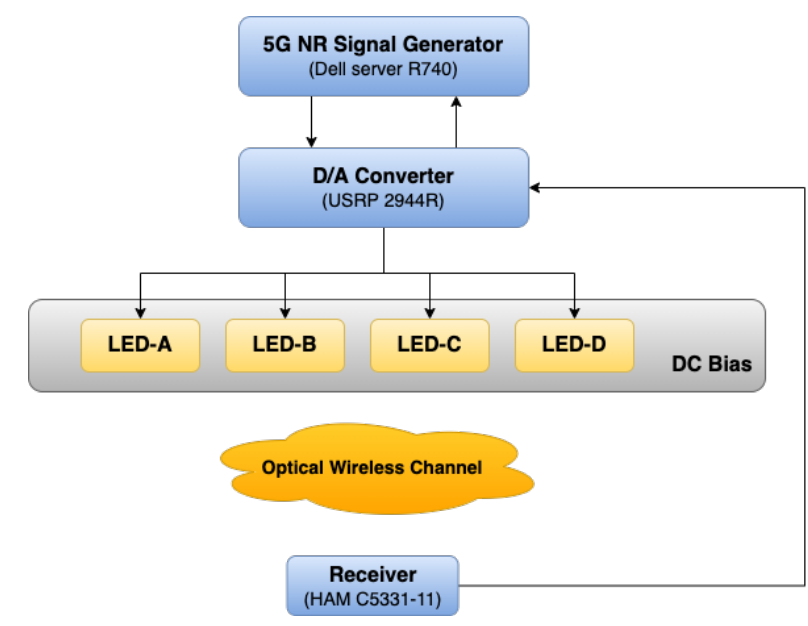

(a) Flow chart of 5G indoor positioning system demonstration

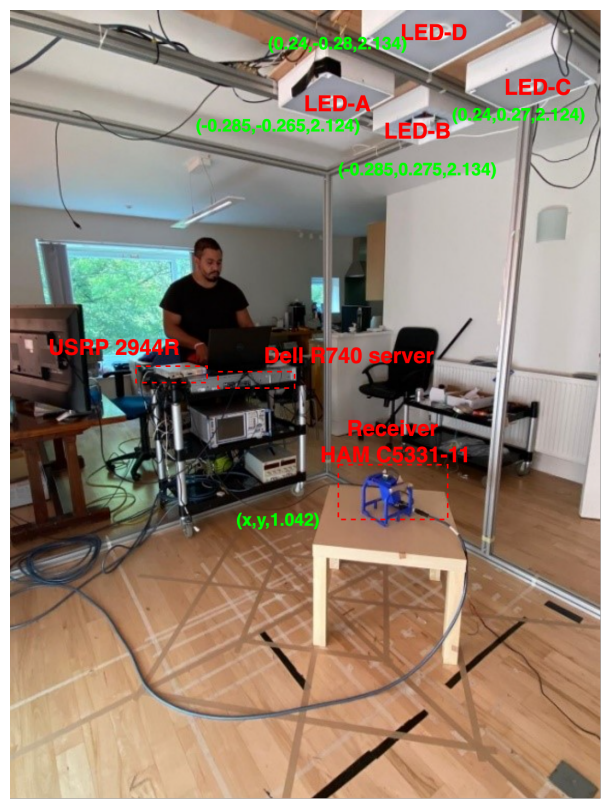

(b) Experimental setup

Fig. 4. 5G indoor positioning system architecture

TABLE I

PARAMETERS OF THE VLP SYSTEM

\begin{tabular}{|c|c|}
\hline Parameter & Value \\
\hline Positioning unit size $(L W H) / m^{3}$ & $0.3 \times 0.3 \times 2.14$ \\
\hline \multirow{3}{*}{ Positions of four LEDs $(x, y, z)(m)$} & LED-A $(-0.285,-0.265,2.124)$ \\
& LED-B $(-0.285,0.275,2.134)$ \\
& LED-C $(0.24,0.27,2.124)$ \\
& LED-D $(0.24,-0.28,2.134)$ \\
\hline Height of the receiver $(m)$ & $0.272,1.042$ \\
\hline Plane range of the receiver $(m)$ & $(-0.3,-0.3)$ to $(0.3,0.3)$ \\
(resolution: 0.1$)$
\end{tabular}




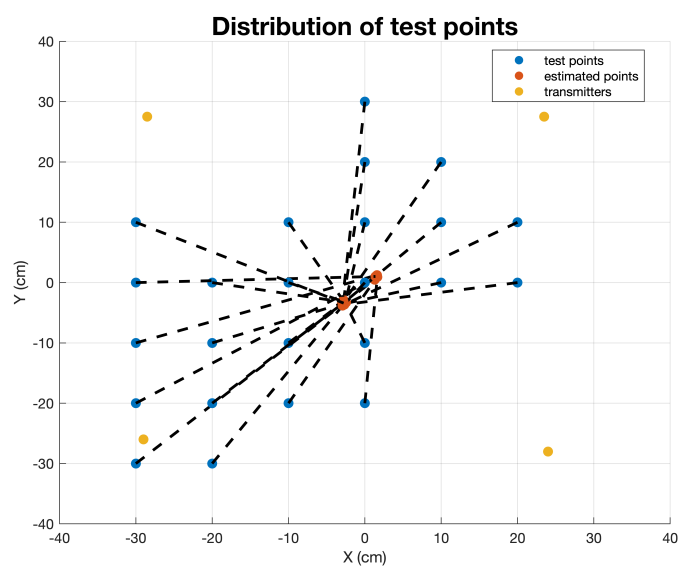

Fig. 5. Distribution of reference points and estimated points of the method without subcarrier extraction

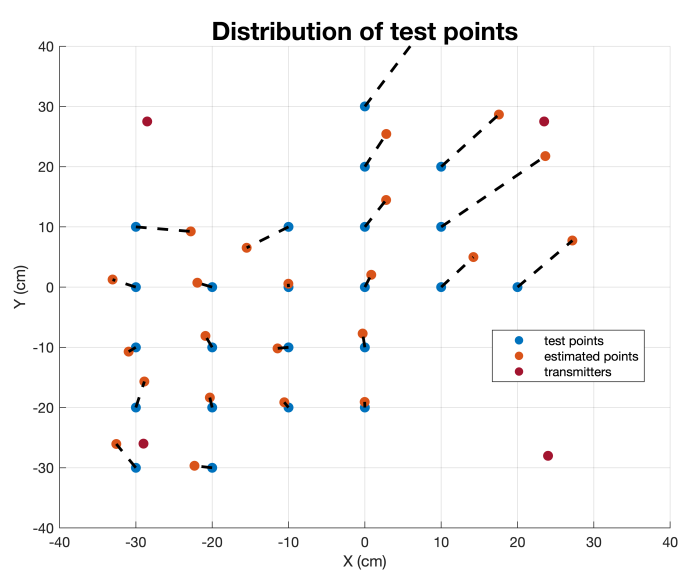

Fig. 6. Distribution of reference points and estimated points of our proposal

it can be shown from the figure that the estimated points were far from their real positions. At the same time, their estimated positions were closer to them at points in the center of the location region, which resulted in a minimum positioning error of $4.10 \mathrm{~cm}$ in $(0,0)$. In contrast, the maximum positioning error occured farthest from the center of the region, reaching $43.68 \mathrm{~cm}$ in $(-30,-30)$. Subcarriers extracted by our proposal in each receiver's reference point are shown in Table.III.

The distribution of reference points and estimated points are shown in Fig.6. As we analyzed and extracted the subcarriers, the average positioning error came to $5.97 \mathrm{~cm}$ and the minimum positioning error achieved $0.55 \mathrm{~cm}$ in $(-10,0)$. The place with the greatest positioning error appeared in $(-20,-30)$, which reached $20.34 \mathrm{~cm}$. Compared with the previous method, our proposal reduces $73.28 \%$ of average positioning error.

Fig. 7 and Fig. 8 respectively show the cumulative distribution function(CDF) of two methods. When we considered 10 $\mathrm{cm}$ as the acceptable positioning error, $48 \%$ of test points met this requirement when using the method without subcarrier extraction. When the subcarriers were extracted by using our

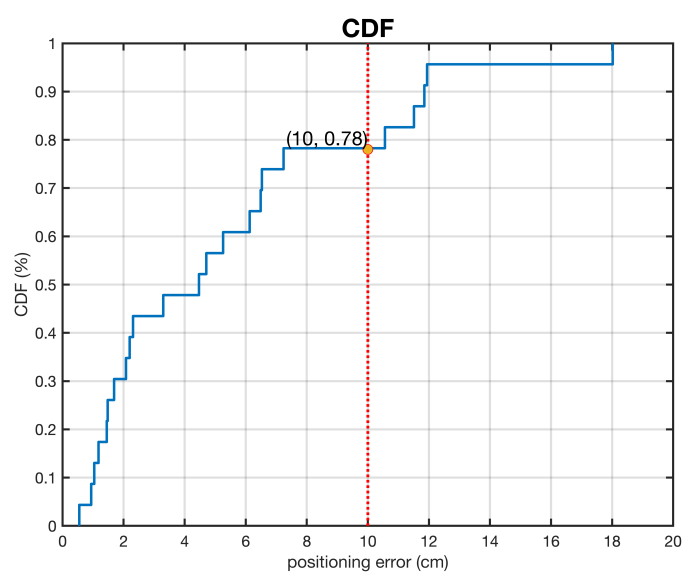

Fig. 7. Cumulative Distribution Function(CDF) when the height of receiver is $0.272 \mathrm{~m}$

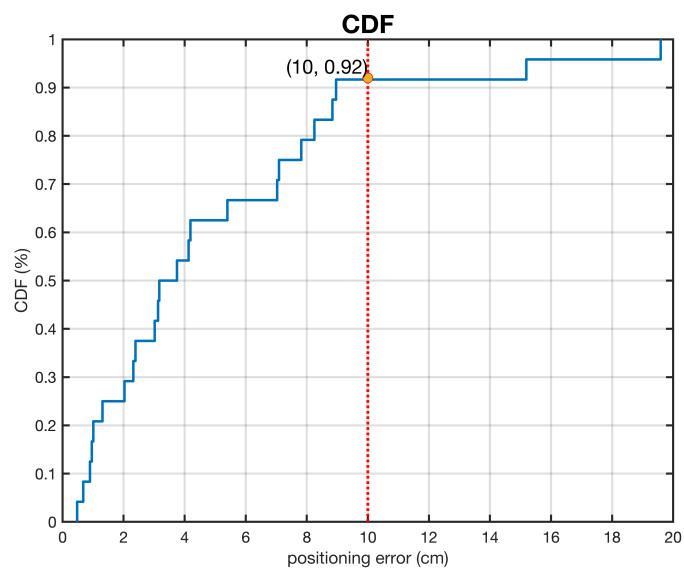

Fig. 8. Dumulative Distribution Function (CDF) when the height of receiver is $1.042 \mathrm{~m}$

proposal, this ratio has increased to $78 \%$. In other words, our proposed method can increase the number of high-precision test points in the positioning area.

\section{CONClusion}

In this paper, we apply a positioning reference signal(PRS) extraction method in $5 \mathrm{G}$ visible light positioning system. By using the particle swarm optimization algorithm, environment indexes describing the current illumination and communication environment were fitted, and successfully extracted the PRS with the least environmental impact for positioning calculations. The experimental results verify that by extracting subcarriers from 5G NR signal sources, the positioning error was reduced by $73.28 \%$. Future work includes improve this algorithm to three-dimensional positioning algorithm and optimize algorithm to adapt to a larger positioning area.

\section{ACKNOWLEDGMENT}

The authors gratefully acknowledge the financial support of the Bourgogne-Franche-Comté Region (Robassist project) and 
TABLE II

POSITIONING ERRORS OF TWO METHODS

\begin{tabular}{|c|c|c|c|}
\hline Positioning method & Accuracy PE (cm) & Minimum PE (cm) & Maximum PE (cm) \\
\hline Method without subcarrier extraction & 22.34 & 4.10 & 43.68 \\
\hline Proposal & 5.97 & 0.55 & 20.34 \\
\hline
\end{tabular}

TABLE III

EXTRACTED SUBCARRIERS OF EACH LED BY OUR PROPOSAL

\begin{tabular}{|c|c|c|c|c|}
\hline Receiver's location(cm) & Subcarrier of LED-A & Subcarrier of LED-B & Subcarrier of LED-C & Subcarrier of LED-D \\
\hline$(-30,-30)$ & 31 & 22 & 14 & 3 \\
\hline$(-30,-20)$ & 9 & 14 & 31 & 2 \\
\hline$(-30,-10)$ & 9 & 4 & 2 & 3 \\
\hline$(-30,0)$ & 11 & 21 & 26 & 5 \\
\hline$(-30,10)$ & 4 & 17 & 6 & 5 \\
\hline$(-20,-30)$ & 2 & 1 & 12 & 10 \\
\hline$(-20,-20)$ & 2 & 10 & 11 & 9 \\
\hline$(-20,0)$ & 5 & 15 & 9 & 4 \\
\hline$(-10,-20)$ & 13 & 30 & 6 & 7 \\
\hline$(-10,-10)$ & 13 & 5 & 1 & 16 \\
\hline$(-10,0)$ & 2 & 14 & 20 & 26 \\
\hline$(-10,10)$ & 8 & 6 & 3 & 16 \\
\hline$(0,-20)$ & 176 & 88 & 12 & 8 \\
\hline$(0,-10)$ & 1 & 3 & 3 & 5 \\
\hline$(0,10)$ & 1 & 28 & 2 & 5 \\
\hline$(0,20)$ & 9 & 13 & 3 & 1 \\
\hline$(0,30)$ & 3 & 9 & 30 & 4 \\
\hline$(10,0)$ & 22 & 14 & 10 & 2 \\
\hline$(10,10)$ & 3 & 3 & 37 & 9 \\
\hline$(20,0)$ & 1 & 7 & 10 & 19 \\
\hline$(20,10)$ & 3 & 3 & 10 & 6 \\
\hline
\end{tabular}

the EU Horizon 2020 program towards the Internet of RadioLight project H2020-ICT 761992.

\section{REFERENCES}

[1] S. Elayoubi and M. Maternia, " $5 \mathrm{~g}$-ppp use cases and performance evaluation modeling," in $5 G$ PPP white paper, 1.0., 5G-PPP project collaboration, vol. 1, p. 81, Wiley, 2016.

[2] L. Shi, W. Li, X. Zhang, Y. Zhang, G. Chen, and A. Vladimirescu, "Experimental 5g new radio integration with vlc," in 2018 25th IEEE International Conference on Electronics, Circuits and Systems (ICECS), pp. 61-64, IEEE, 2018.

[3] L. Shi, X. Zhang, A. Vladimirescu, Z. Wang, Y. Zhang, J. Wang, J. Garcia, J. Cosmas, and A. Kapovits, "Experimental testbed for VLC-based localization framework in 5G Internet of Radio Light," in 2019 26th IEEE International Conference on Electronics, Circuits and Systems, ICECS 2019, pp. 430-433, Institute of Electrical and Electronics Engineers Inc., nov 2019.

[4] K. Cabaj, M. Gregorczyk, W. Mazurczyk, P. Nowakowski, and P. Zórawski, "Network Threats Mitigation Using Software-Defined Networking for the 5G Internet of Radio Light System," Security and Communication Networks, vol. 2019, 2019.

[5] J. Luo, L. Fan, and H. Li, "Indoor Positioning Systems Based on Visible Light Communication: State of the Art," IEEE Communications Surveys and Tutorials, vol. 19, no. 4, pp. 2871-2893, 2017.

[6] C. Huang, X. Zhang, F. Zhou, Z. Wang, and L. Shi, "NLOS-Aware VLC-based Indoor Localization: Algorithm Design and Experimental Validation," in IEEE Wireless Communications and Networking Conference, WCNC, vol. 2020-May, Institute of Electrical and Electronics Engineers Inc., may 2020.

[7] Y. H. Hsueh, P. C. Su, Y. F. Lin, H. H. Huang, and T. C. Liu, "Robot indoor navigation using visible light communication," Proceedings of the
2018 Pacific Neighborhood Consortium Annual Conference and Joint Meetings: Human Rights in Cyberspace, PNC 2018, pp. 1-6, 2018.

[8] H. Zhang, J. Cui, L. Feng, A. Yang, H. Lv, B. Lin, and H. Huang, "HighPrecision Indoor Visible Light Positioning Using Modified Momentum Back Propagation Neural Network with Sparse Training Point," Sensors, vol. 19, p. 2324, may 2019.

[9] L. Shi, D. Shi, X. Zhang, B. Meunier, H. Zhang, Z. Wang, A. Vladimirescu, W. Li, Y. Zhang, J. Cosmas, K. Ali, N. Jawad, R. Zetik, E. Legale, M. Satta, J. Wang, and J. Song, "5G Internet of Radio Light Positioning System for Indoor Broadcasting Service," in IEEE Transactions on Broadcasting, vol. 66, pp. 534-544, Institute of Electrical and Electronics Engineers Inc., jun 2020.

[10] B. El Boudani, L. Kanaris, A. Kokkinis, M. Kyriacou, C. Chrysoulas, S. Stavrou, and T. Dagiuklas, "Implementing Deep Learning Techniques in 5G IoT Networks for 3D Indoor Positioning: DELTA (DeEp LearningBased Co-operaTive Architecture)," Sensors, vol. 20, p. 5495, sep 2020.

[11] Z. Deng, X. Zheng, C. Zhang, H. Wang, L. Yin, and W. Liu, "A TDOA and PDR Fusion Method for 5G Indoor Localization Based on Virtual Base Stations in Unknown Areas," IEEE Access, vol. 8, pp. 225123 225133, 2020.

[12] H. Yang, W. D. Zhong, C. Chen, and A. Alphones, "Integration of Visible Light Communication and Positioning within 5G Networks for Internet of Things," IEEE Network, vol. 34, pp. 134-140, sep 2020.

[13] D. Shi, X. Zhang, L. Shi, A. Vladimirescu, W. Mazurczyk, K. Cabaj, B. Meunier, K. Ali, J. Cosmas, and Y. Zhang, "On Improving 5G Internet of Radio Light Security Based on LED Fingerprint Identification Method," Sensors, vol. 21, p. 1515, feb 2021.

[14] D. Shi, J. Li, Y. Liu, L. Shi, Y. Huang, Z. Wang, X. Zhang, A. Vladimirescu, and al Effect, "Effect of Illumination Intensity on LED Based Visible Light Communication System," pp. 1-4, 2020.

[15] Y. Liu and Y. F. Zheng, "One-against-all multi-class SVM classification using reliability measures," in Proceedings of the International Joint Conference on Neural Networks, vol. 2, pp. 849-854, 2005. 